PROGRAMA DE INICIAÇÃO CIENTÍFICA

AVALIAÇÃO DO CRESCIMENTO DE TILÁPIAS PRODUZIDAS EM TANQUES DE FERROCIMENTO COM RENOVAÇÃO PARCIAL DE ÁGUA E AERAÇÃO FORÇADA 


\title{
AVALIAÇÃO DO CRESCIMENTO DE TILÁPIAS PRODUZIDAS EM TANQUES DE FERROCIMENTO COM RENOVAÇÃO PARCIAL DE ÁGUA E AERAÇÃO FORÇADA
}

\author{
Relatório final de pesquisa de Iniciação Científica \\ apresentado à Assessoria de Pós-Graduação e \\ Pesquisa pela Faculdade de Ciências da \\ Educação e da Saúde - FACES. \\ Orientação: Carlos Alberto da Cruz Júnior
}




\title{
AVALIAÇÃO DO CRESCIMENTO DE TILÁPIAS PRODUZIDAS EM TANQUES DE FERROCIMENTO COM RENOVAÇÃO PARCIAL DE ÁGUA E AERAÇÃO FORÇADA
}

\author{
Ana Luiza Alves Panta Vasconcelos - UniCEUB, PIC Institucional, bolsista \\ analuizavasconcelos14@gmail.com
}

\begin{abstract}
Carlos Alberto da Cruz Junior - UniCEUB, professor orientador carlos.junior@uniceub.br
\end{abstract}

Manoel Maciel da Costa - UnB, aluno voluntário neto.dad@hotmail.com

\section{Bernardo Ramos Simões Corrêa - UnB, pesquisador colaborador bernersc@hotmail.com}

Grande parte da população mundial vulnerável sobrevive em regiões com escassez hídrica e solos distróficos, demandando de forma emergencial sistemas de produção sustentáveis, participativos e que garantam a segurança alimentar. A tecnologia social de ferrocimento tem sido utilizada no Brasil principalmente para o armazenamento de água da chuva na região nordeste, entretanto apresenta características construtivas que permitem o seu uso na produção de peixes. $O$ presente estudo foi realizado na Estação Experimental de Agroecologia UniCEUB, Chácara Delfim T61, situada no Córrego do Urubu, Lago Norte, Distrito Federal, com o objetivo de avaliar o crescimento de tilápia do Nilo (Oreochromis niloticus) em tanques de ferrocimento com baixo consumo de água. Foram utilizados três tanques de $12 \mathrm{~m}^{3} \mathrm{cada}$, com sistema de aeração forçada por soprador difusor de $1,5 \mathrm{HP}$ e renovação diária da água na ordem de $30 \%$ do volume total de cada tanque. $O$ experimento foi realizado durante 4 meses, de março a junho de 2017. O primeiro tanque foi povoado com 1000 alevinos e após dois meses de crescimento da população de peixes, foram coletados de forma aleatória 300 animais para povoamento (repicagem) dos outros dois tanques, permanecendo nesse sistema por mais dois meses. Foram realizadas morfometrias no povoamento, após 60 dias do povoamento e aos 120 dias e os parâmetros de qualidade de água foram avaliados 2 vezes por semana. As variáveis de qualidade da água (oxigênio dissolvido $(\mathrm{mg} / \mathrm{L}), \mathrm{pH}$, transparência $(\mathrm{cm})$ e nitrito $(\mathrm{ppm})$ ) não apresentaram diferenças estatísticas significativas durante o experimento e mantiveram-se nas faixas ideais para o cultivo da espécie demonstrando a estabilidade do sistema proposto e capacidade do sistema soprador difusor em aerar a coluna d'água. $\mathrm{O} \mathrm{pH}$ apresentou valor médio de 8,2 . Foi verificado redução da temperatura média em $5^{\circ} \mathrm{C}$ no período de dois meses após a repicagem. Na fase pós-repicagem os níveis de amônia tóxica diminuíram demonstrando atividade biológica no sistema. Todos os parâmetros morfológicos levantados, peso (g), comprimento total $(\mathrm{cm})$, comprimento sem cauda $(\mathrm{cm})$ e comprimento de dorso $(\mathrm{cm})$ apresentaram diferenças estatísticas significativas pelo teste de Tuckey $(p<0,01)$ nas três análises realizadas, indicando o crescimento dos animais. Os resultados 
demonstram que apesar de ter ocorrido queda na temperatura da água em virtude das baixas temperaturas do ambiente e o $\mathrm{pH}$ ter valor médio no limite máximo aceitável para a espécie, o sistema de produção em tanque de ferrocimento com baixo consumo de água e aeração forçada permite o crescimento satisfatório de tilápias.

Palavras-chave: Aerador. Peixe. Tecnologia Social. Segurança Alimentar. 


\section{Sumário}

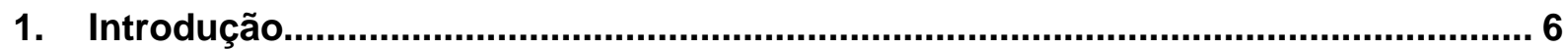

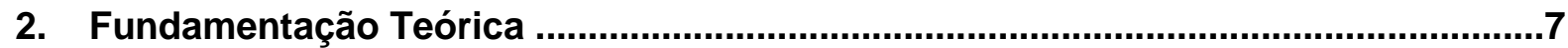

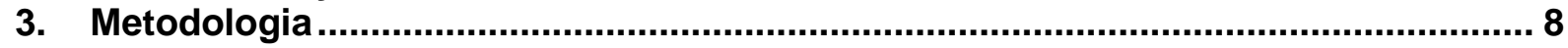

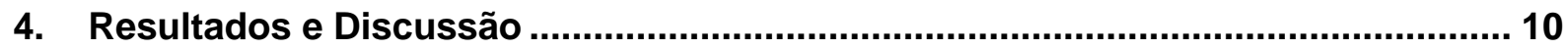

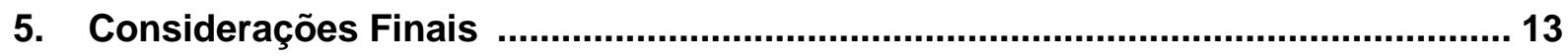

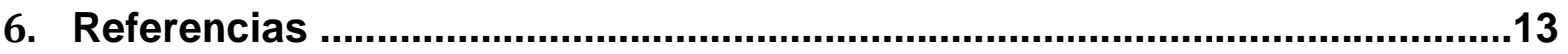




\section{Introdução}

A aquicultura vem assumindo importância cada vez maior no panorama do abastecimento alimentar, sendo o setor da produção animal que mais cresce a uma taxa anual maior que o da população $(6,6 \%)$, ocorrendo o aumento da atividade de 0,7\% em 1970 para 7,8\% em 2008 (FAO, 2010). Em 2011, a produção mundial de pescados foi de 38.984 .542 toneladas (FAO, 2013). A produção aquícola brasileira em 2011 foi de 628.704 toneladas, representando um incremento de $31,1 \%$ em relação à produção de 2010 (MPA, 2011). Comparando-se a produção atual com o montante produzido em 2009 (415.649,0 t), fica evidente o crescimento do setor no país, com um incremento de 51,2\% na produção durante o triênio 2009-2011(MPA, 2011). Seguindo o padrão observado nos anos anteriores, a maior parcela da produção aquícola é oriunda da aquicultura continental, na qual se destaca a piscicultura representando $86,6 \%$ da produção total nacional.

A tilápia do Nilo (Oreochromis niloticus) faz parte de um grupo de espécies que recebem a denominação genérica de tilápias, pertence à família Cichlidae (Perciformes) e que têm a sua origem no continente africano. No Brasil, a espécie foi introduzida no ano de 1971, proveniente da África, inicialmente na região nordeste e a partir de então distribuída pelo país. Apresenta-se hoje como uma das espécies mais importantes para o desenvolvimento da aquicultura nacional e mundial, uma vez que é a espécie mais produzida no país, responsável por $39,4 \%$ da produção da aquicultura de água doce (MPA, 2012). Além disso, compõe o segundo grupo de espécies mais cultivadas em todo o mundo, ficando atrás apenas das carpas. Este destaque devesse, em grande parte, às características apresentadas por estes peixes, como rápido crescimento, tolerância a uma ampla faixa de condições ambientais, precocidade sexual, rusticidade e capacidade de aproveitamento do alimento natural (EL-SAYED, 2006). Possui ainda boas características organolépticas e ausência de espinhos na forma de Y no seu filé (HILDSORF, 1995).

O hábito alimentar da tilápia do Nilo é onívoro (SKLAN et al., 2004), fato este que a coloca em vantagem em relação a outras espécies que demandam de grande quantidade de farinha de peixe nas dietas como fonte de proteínas (FITZSIMMONS, 2000). Esta vantagem reside principalmente na possibilidade de assimilar eficientemente os carboidratos presentes nos ingredientes de origem vegetal das 
rações (TEIXEIRA et al.,2008). Quando nas fases iniciais de vida, são capazes de utilizar alimentos artificiais imediatamente após a absorção do saco vitelino (ELSAYED, 2006). As características apresentadas anteriormente tornam-se de alta relevância no contexto da produção comercial, que passa por um momento de crescimento e intensificação dos cultivos, associados a uma demanda de consumidores cada vez mais exigentes quanto às questões ambientais (LITTLE et al., 2008).

Entre as opções de criação, o sistema intensivo em tanques de ferrocimento permite densidades elevadas, desde que haja a aplicação de tecnologias apropriadas, tais como: o monitoramento da qualidade da água, aeração forçada e o uso adequado da ração. Vale ressaltar que o sistema pode ser construído com materiais e mão de obra local em pequenas áreas e com baixo custo de implantação. Outra vantagem é que o tanque é construído sobre o solo, sendo possível a construção em qualquer época do ano, diferentemente dos tanques de terra, que devem ser construídos na época seca.

Diante das vantagens de se utilizar o tanque de ferrocimento na aquicultura e a escassez de informações relativas ao cultivo, o presente projeto teve por objetivo avaliar o crescimento de tilápias da linhagem GIFT em tanques de ferrocimento com renovação de água (30\% dia) e aeração forçada.

\section{Fundamentação Teórica}

A espécie de peixe mais produzida no Brasil é a tilápia do Nilo (MPA, 2011). Em 2011 foram produzidas 253.824 toneladas, representando $46 \%$ do total de pescado (MPA, 2011). Trata-se de uma espécie rústica, muito resistente, de crescimento rápido e hábito alimentar onívoro (BEYRUTH et al., 2004). Está entre as espécies que possuem características preferidas pela aquicultura, por terem boa aceitação e elevado valor comercial no mercado consumidor (ZIMMERMANN \& HASPER, 2003).

Os sistemas mais comuns para o cultivo de tilápias são a produção em viveiros escavados ou em tanques-rede instalados em reservatórios de domínio público. A produtividade em viveiros varia de 8 a 10 ton/ha/ciclo e em tanques rede de 80 a $120 \mathrm{~kg} / \mathrm{m}^{3}$, sendo o peso médio $(600 \mathrm{~g})$ alcançado em um período de 180 dias, a partir de alevinos de 0,5 gramas (KUBITZA, 2011). 
Uma piscicultura intensiva consiste geralmente em estocar certo número de alevinos, com aproximadamente a mesma idade em um viveiro, e despescá-los depois de certo tempo. Periodicamente (em intervalos constantes de tempo), pode-se obter através de amostras, o comprimento médio (Lt) e o peso médio ( $\mathrm{Wt}$ ), dos indivíduos em cada período do cultivo, bem como a mortalidade (SANTOS, 1978), e assim realizar estimativas da produção. No intuito de quantificar o crescimento das espécies utilizadas nos cultivos, alguns trabalhos são encontrados. Alcantara-Filho \& Makrakis (1994) em um ensaio com a pirapitinga (Colossoma brachypomus), estimaram e analisaram as curvas de biomassa total, simples e acumulada, sobrevivência e mortalidade, em viveiros escavados no Centro de Pesquisas Ictiológicas "Rodolpho von lhering" do DNOCS.

Carmo (2003) realizou estudos sobre o crescimento de três linhagens de tilápia (Bouaké, Vermelha e Chitralada), criadas em viveiros com sistema semiintensivo na Estação de Aquicultura Continental Prof. Johei Koike, do Departamento de Pesca, da Universidade Federal Rural de Pernambuco (UFRPE) Recife-PE-Brasil, no período de dezembro de 2002 a março de 2003. Em um estudo para avaliar o crescimento morfométrico e alométrico de linhagens de tilápia (Oreochromis niloticus).

Santos (2004) utilizou as linhagens Chitralada e Genomar Supreme, estabelecendo as curvas de crescimento morfométrico e alométrico, os rendimentos do processamento dos peixes em diferentes pesos de abate e definiu as medidas morfométricas necessárias para a classificação de carcaças de tilápias.

Em um estudo com tilápia do Nilo, criadas em tanques-rede no reservatório Jurumirim (Rio Paranapanema), Landell (2007) avaliou o desempenho produtivo da linhagem Chitralada, através das curvas de crescimento e biomassa, e ainda a sobrevivência e mortalidade entre os meses de dezembro de 2004 a setembro de 2005. Porém, são escassos estudos para verificar a eficiências produtiva de tilápias produzidas em sistema intensivo de ferrocimento, no sentido de estabelecer os parâmetros de crescimento e a curva de biomassa.

\section{Metodologia}

O experimento foi conduzido em fase única de cultivo, no período de março a julho de 2017, na Estação Experimental de Agroecologia - UniCEUB, Chácara Delfim 
T61, situada no Córrego do Urubu, Lago Norte, Distrito Federal. Foram utilizados 3 tanques de ferrocimento de $12 \mathrm{~m}^{3}$ cada, com sistema de aeração forçada por soprador difusor de 1,5 HP e renovação diária da água na ordem de $30 \%$ do volume total de cada tanque. $\mathrm{O}$ abastecimento dos tanques foi realizado por gravidade. $\mathrm{O}$ povoamento dos tanques com Oreochormis nicloticus da variedade gift, monosexo machos, foi realizado após sete dias do enchimento, na densidade de 100 peixes $\mathrm{m}^{3}$ com peso médio inicial de $6 \mathrm{~g}$ (malha 12). Todos os animais utilizados foram do mesmo lote e provenientes de fornecedor idôneo.

O primeiro tanque foi povoado com 1000 alevinos e após dois meses de crescimento da população de peixes, foram coletados ao acaso 300 animais para povoamento (repicagem) dos outros dois tanques, permanecendo nesse sistema por mais dois meses. Foram realizadas morfometrias (peso $(\mathrm{g})$, comprimento total $(\mathrm{cm})$, comprimento sem cauda $(\mathrm{cm})$ e comprimento de dorso $(\mathrm{cm}))$ no povoamento, após 60 dias do povoamento e aos 120 dias, e os parâmetros de qualidade de água foram avaliados 2 vezes por semana.

Os peixes foram alimentados 4 vezes ao dia (fase de alevino e juvenil) e quatro vezes ao dia (fase adulta), com ração comercial do tipo farelada para alevinos, e extrusada para juvenil e adulto (36\% e $32 \%$ de proteína bruta). A ração foi ofertada variando de 4 a $2 \%$ da biomassa contida em cada tanque (KUBITZA, 2006). Para obtenção da biomassa e para o ajuste da quantidade de ração fornecida, os animais foram pesados nas biometrias em balança de precisão de $0,01 \mathrm{~g}$ e devolvidos para seus respectivos tanques.

A qualidade da água de abastecimento e de cada tanque foi monitorada diariamente pela manhã e à tarde sempre nos mesmos horários. Analisou-se temperatura, $\mathrm{pH}$, oxigênio dissolvido e condutividade elétrica por meio de sonda multiparâmetros e a amônia e nitrito por meio de Kits comerciais para piscicultura. Já a transparência, indicativo da concentração da população de plâncton ou a suspensão de sedimentos finos, com o disco de Secchi.

Para a análise estatística foi aplicado o teste de Variância (ANOVA-one way) para detectar diferenças $(\mathrm{P}<0,05)$ entre as médias. Posteriormente utilizou-se o teste de Tuckey ( $5 \%$ de probabilidade) para a comparação de médias. Os testes estatísticos foram realizados no programa Assistat ${ }^{\circledR}$. 


\section{Resultados e Discussão}

Alguns autores como Lennard (2004) e Braz (2000), afirmam que as crescentes restrições e custos quanto ao uso da água já obrigam produtores rurais em inúmeros países a buscarem alternativas mais econômicas a respeito da utilização da água para viabilização da produção de alimentos. Diante desse cenário, a piscicultura em tanques de ferrocimento configura-se como uma atividade que vem crescendo no Brasil, principalmente na região Nordeste e Centro-oeste. Do ponto de vista teórico, apresenta vantagens técnicas, ecológicas e sociais sobre o extrativismo e a piscicultura tradicional em viveiros de terra, sendo uma técnica relativamente barata e simples, quando comparada com a tradicional.

A tilápia, por apresentar características de alta relevância no contexto da produção comercial, é hoje uma das espécies mais produzidas no país (MPA, 2012), e teve bom desenvolvimento no sistema de ferrocimento proposto.

Durante o projeto foram realizadas análises da qualidade da água e os resultados são apresentados na tabela 1. 
Tabela 1 - Resultado dos parâmetros de água analisados durante 0 experimento nas três fases do cultivo.

\begin{tabular}{|c|c|c|c|c|c|c|}
\hline & FASE & MÉDIA & $\begin{array}{l}\text { DESVIO } \\
\text { PADRÃO }\end{array}$ & $\begin{array}{c}\text { Coeficientes } \\
\text { de Variação } \\
\%\end{array}$ & MENOR VALOR & MAIOR VALOR \\
\hline \multirow{3}{*}{$\begin{array}{l}\text { Oxigênio Dissolvido } \\
\text { (mg/L) }\end{array}$} & 1 & $5,44 a$ & 2,28 & 41,49 & 3,32 & 10,22 \\
\hline & 2 & $6,43 a$ & 2,00 & 31,15 & 3,04 & 9,40 \\
\hline & 3 & $6,83 a$ & 2,18 & 31,92 & 3,45 & 11,58 \\
\hline \multirow{3}{*}{ Temperatura $\left({ }^{\circ} \mathrm{C}\right)$} & 1 & $26,66 a$ & 1,64 & 6,16 & 24,00 & 30,30 \\
\hline & 2 & $21,72 b$ & 2,65 & 12,21 & 17,40 & 26,70 \\
\hline & 3 & $21,49 b$ & 2,40 & 11,15 & 17,30 & 25,70 \\
\hline \multirow{3}{*}{$\mathrm{pH}$} & 1 & $8,24 a$ & 0,55 & 6,67 & 7,30 & 9,31 \\
\hline & 2 & $8,23 a$ & 0,59 & 7,16 & 7,29 & 9,73 \\
\hline & 3 & $8,36 a$ & 0,49 & 5,84 & 7,51 & 9,47 \\
\hline \multirow{3}{*}{ Transparência (cm) } & 1 & $39,64 a$ & 10,57 & 26,67 & 24,00 & 60,00 \\
\hline & 2 & $35,55 a$ & 6,62 & 18,63 & 23,00 & 49,00 \\
\hline & 3 & $35,90 a$ & 6,46 & 17,99 & 27,00 & 54,00 \\
\hline \multirow{3}{*}{ Nitrito (ppm) } & 1 & $0,09 a$ & 0,12 & 134,15 & 0,00 & 0,25 \\
\hline & 2 & $0,14 a$ & 0,15 & 107,18 & 0,00 & 0,50 \\
\hline & 3 & $0,28 a$ & 0,43 & 154,55 & 0,00 & 1,75 \\
\hline \multirow{3}{*}{$\begin{array}{c}\text { Amônia Tóxica } \\
\text { (ppm) }\end{array}$} & 1 & $0,13 a$ & 0,12 & 83,36 & 0,00 & 0,44 \\
\hline & 2 & $0,03 b$ & 0,03 & 113,73 & 0,00 & 0,13 \\
\hline & 3 & $0,04 b$ & 0,06 & 137,52 & 0,00 & 0,26 \\
\hline
\end{tabular}

As variáveis de qualidade da água (oxigênio dissolvido (mg/L), $\mathrm{pH}$, transparência $(\mathrm{cm})$ e nitrito $(\mathrm{ppm})$ ) não apresentaram diferenças estatísticas significativas durante o experimento e mantiveram-se nas faixas ideais para o cultivo da espécie demonstrando a estabilidade do sistema proposto e capacidade do sistema soprador difusor em aerar a coluna d'água.

$\mathrm{O} \mathrm{pH}$ apresentou valor médio de 8,2 e foi verificado redução da temperatura média em $5^{\circ} \mathrm{C}$ no período de dois meses após a repicagem. Na fase pós-repicagem os níveis de amônia tóxica diminuíram demonstrando atividade biológica no sistema. 
Todos os parâmetros morfométricos levantados (tabela 2), peso (g), comprimento total $(\mathrm{cm})$, comprimento sem cauda $(\mathrm{cm})$ e comprimento de dorso $(\mathrm{cm})$ apresentaram diferenças estatísticas significativas pelo teste de Tuckey $(p<0,01)$ nas três análises realizadas, indicando o crescimento dos animais.

Tabela 2 - Resultado das variáveis morfométricas analisadas durante o experimento nas três fases do cultivo.

\begin{tabular}{ccccccc}
\hline & FASE & $\mathrm{X}$ & $\mathrm{DP}$ & $\mathrm{CV} \%$ & MENOR VALOR & MAIOR VALOR \\
\hline \multirow{2}{*}{ PESO $(\mathrm{g})$} & 1 & $4,92 \mathrm{c}$ & 0,80 & 16,35 & 4,00 & 6,00 \\
& 2 & $52,52 \mathrm{~b}$ & 12,30 & 23,42 & 40,00 & 90,00 \\
& 3 & $136,35 \mathrm{c}$ & 30,00 & 22,00 & 100,00 & 223,00 \\
\hline \multirow{2}{*}{ Comprimento } & 1 & $6,32 \mathrm{c}$ & 0,44 & 6,90 & 5,50 & 7,20 \\
Total $(\mathrm{cm})$ & 2 & $15,55 \mathrm{~b}$ & 1,04 & 7,47 & 12,60 & 16,90 \\
& 3 & $19,00 \mathrm{a}$ & 1,31 & 6,90 & 16,70 & 22,50 \\
\hline \multirow{2}{*}{ Comprimento } & 1 & $5,10 \mathrm{c}$ & 0,32 & 6,38 & 4,40 & 5,80 \\
sem cauda (cm) & 2 & $11,38 \mathrm{~b}$ & 0,95 & 8,39 & 10,20 & 14,90 \\
& 3 & $15,40 \mathrm{a}$ & 1,10 & 7,05 & 13,50 & 19,50 \\
\hline \multirow{2}{*}{ Comprimento } & 1 & $1,94 \mathrm{c}$ & 0,12 & 6,31 & 1,70 & 2,20 \\
Dorso $(\mathrm{cm})$ & 2 & $4,55 \mathrm{~b}$ & 0,33 & 7,19 & 3,90 & 5,30 \\
& 3 & $6,87 \mathrm{a}$ & 0,57 & 8,28 & 5,80 & 8,30 \\
\hline
\end{tabular}

Os resultados demonstram que apesar de ter ocorrido queda na temperatura da água em virtude das baixas temperaturas do ambiente e o $\mathrm{pH}$ ter valor médio no limite máximo aceitável para a espécie, o sistema de produção em tanque de ferrocimento com baixo consumo de água e aeração forçada permitiu o crescimento satisfatório de tilápias dentro do sistema proposto.

\section{Considerações Finais}

O sistema demonstra ser uma opção viável para pequenos agricultores uma vez que a implantação, a operação e o monitoramento são fáceis de executar e caracteriza-se com um sistema inovador que permite reduzir o consumo da água, elimine desperdícios e promover o reuso e a reciclagem 
Novos estudos devem ser elaborados para encontrar a melhor densidade para a produção sustentável e ao mesmo tempo para o conforto animal.

\section{Referências}

BRAZ, M.. Qualidade na produção de peixes em sistemas de recirculação de água. Centro Universitário Nove de

Julho, São Paulo, SP. 2000.

CARMO, J. L. Avaliação do crescimento de três linhagens de Tilápia do gênero Oreochromis sp., em sistema semi-intensivo, cultivadas em viveiros. 62 p. Dissertação (Mestrado em Recursos Pesqueiros e Aqüicultura) Universidade Federal Rural de Pernambuco, Recife, PE. 2003

EL-SAYED, A-F.M. Tilapia culture. CABI Publishing, Oxfordshire, U.K., 2006, 277 pp.

FAO (2010). The State of World Fisheries and Aquaculture. Rome.

FAO (2013). Fishstat Plus (v, 2.32) emitido em 27.01.2014. Food and Agriculture Organization, Rome, Italy.

FITZSIMMONS, K. Tilapia aquaculture in Mexico. In: COSTA-PIERCE, B.A.; RAKOCY, J.E. (Ed.). Tilapia Aquaculture in the Americas, Vol. 2. Baton Rouge, Louisiana, World Aquaculture Society, 2000. p. 171-183.

HILDSORF, A.W.S. Genética e cultivo de tilápias vermelhas, uma revisão. Boletim do Instituto de Pesca, São Paulo,v.22, n.1, p. 73-78, 1995.

KUBITZA, F. (2011) O status atual e as tendências da tilapicultura no Brasil. Revista Panorama da Aquicultura., vol 21, no124. Março/Abril.

LANDELL, M. C. Avaliação do desempenho de tilápias (Oreochromis niloticus) em tanques-rede na represa de Jurumirim/Alto Rio Paranapanema. Dissertação (Mestrado) - Faculdade de Ciências Agrárias e Veterinárias, Universidade Estadual Paulista, Jaboticabal. 2007.

LENNARD, W. A. Aquaponics research at RMIT university, Melbourne Australia. Aquaponics Journal, v. 35, p. p18-24,2004.

LITTLE, D.C.; MURRAY, J.F.; AZIM, M.E.; et al. Warm-water fish production in the UK: limits to green growth? Trends in Food Science and Technology, v. 19, p. $255 \square 264,2008$.

MINISTÉRIO DA PESCA E AQUICULTURA - MPA. (2011) Boletim Estatistico da Pesca e Aquicultura 2011. Disponível em http: // www.mpa.gov.br . acesso em: 19 agost. 2017. 
MINISTÉRIO DA PESCA E AQUICULTURA- MPA. (2012). Boletim Estatístico da Pesca e Aquicultura - Brasil 2010.

Ministério da Pesca e Aquicultura, Brasília, DF, 2012. 128p.

SANTOS, E. P. Dinâmica de populações aplicada a pesca e piscicultura. Hucitec, São Paulo, SP, p. 13-44, 1978.

SANTOS, VANDER BRUNO. Crescimento morfométrico e alométrico de linhagens de tilápia (Oreochromis niloticus). 86 p. Dissertação (Mestrado em Zootecnia), Universidade Federal de Lavras, Lavras, MG. 2004.

SKLAN, D.; PRAG, T.; LUPATSCH, I. Structure and function of the small intestine of the tilapia Oreochromis niloticus $x$ Oreochromis aureus (Teleostei, Cichlidae). Aquaculture Research, v.35, p.350-357, 2004.

TEIXEIRA, E.A.; CREPALDI, D.V.; FARIA, P.M.C., et al. Composição corporal e exigências nutricionais de aminoácidos para alevinos de tilápia (Oreochromis sp.). Revista Brasileira de Saúde e Produção Animal, v. 9, p. 239-246, 2008. 